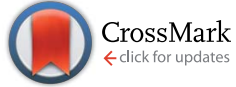

Cite this: Chem. Sci., 2016, 7, 5753

Received 29th March 2016 Accepted 11th June 2016

DOI: $10.1039 / \mathrm{c} 6 \mathrm{sc} 01380 \mathrm{e}$

www.rsc.org/chemicalscience

\section{Embedding electron-deficient nitrogen atoms in polymer backbone towards high performance $n$ - type polymer field-effect transistors $\uparrow$}

\author{
Ya-Zhong Dai, Na Ai, Yang Lu, Yu-Qing Zheng, Jin-Hu Dou, Ke Shi, Ting Lei, \\ Jie-Yu Wang and Jian Pei
}

With $\mathrm{sp}^{2}$-nitrogen atoms embedded in an isatin unit, a donor-acceptor ( $\left.D-A\right)$ conjugated polymer AzaBDOPV-2T was developed with a low LUMO level down to $-4.37 \mathrm{eV}$. The lowered LUMO level as well as the conformation-locked planar backbone provide AzaBDOPV-2T with electron mobilities over $3.22 \mathrm{~cm}^{2} \mathrm{~V}^{-1} \mathrm{~s}^{-1}$ tested under ambient conditions, which is among the highest in $\mathrm{n}$-type polymer fieldeffect transistors (FETs). Our results demonstrate that embedding electron-deficient $\mathrm{sp}^{2}$-nitrogen in conjugated backbones is an effective approach to develop $n$-type polymer semiconductors with high performance.
Conjugated polymers have attracted great interests in low-cost, flexible, and large-area electronic applications due to their solution-processability, good mechanical properties, and tunable electronic properties. ${ }^{1}$ In the past few years, the development of novel building blocks for conjugated polymers, such as benzothiadiazole (BT), ${ }^{2}$ diketopyrrolopyrrole (DPP), ${ }^{3}$ isoindigo (II), ${ }^{4}$ and naphthalene diimide (NDI), ${ }^{5}$ has led to significant progress in the carrier mobilities of polymer semiconductors. Nevertheless, only a few of these polymers can exhibit high electron mobilities when operated under ambient conditions, ${ }^{5 c}$ thus limiting their application. It has been an intriguing research topic to develop high-performance airstable n-type polymer semiconductors in organic electronics.

Low LUMO levels are indeed desirable to facilitate electron injection and enhance the electrochemical stability of n-type polymer semiconductors. ${ }^{6}$ The introduction of electron-withdrawing groups (such as $\mathrm{F}$ or $\mathrm{Cl}$ ) onto polymer conjugated backbones has been shown to be an effective way to lower their LUMO energy levels, thus leading to enhanced n-type transport. ${ }^{6 c, d}$ However, compared to the extensive efforts spent on halogen substitution, embedding electron-deficient $\mathrm{sp}^{2}$ nitrogen atoms in polymer backbones has been somewhat overlooked. ${ }^{7}$ Incorporation of electron-deficient pyridine units into polymer backbones could also lower the energy levels of the resultant conjugated polymers. In addition, the replacement of

Beijing National Laboratory for Molecular Sciences, Key Laboratory of Bioorganic Chemistry and Molecular Engineering of Ministry of Education, Key Laboratory of Polymer Chemistry and Physics of Ministry of Education, Center for Soft Matter Science and Engineering, College of Chemistry and Molecular Engineering, Peking University, Beijing 100871, China. E-mail: jianpei@pku.edu.cn

$\dagger$ Electronic supplementary information (ESI) available. See DOI: 10.1039/c6sc01380e benzene rings with pyridine units is also beneficial to improving the planarity of polymer backbones because $\mathrm{sp}^{2}$-nitrogen atoms are less sterically demanding than $\mathrm{CH}$ units. ${ }^{8}$ Therefore, such a $\mathrm{sp}^{2}$-nitrogen embedding strategy may provide new opportunities to develop decent $n$-type semiconductors.

Recently, benzodifurandione-based oligo( $p$-phenylene vinylene) (BDOPV), which has four electron-withdrawing carbonyl groups on the backbone and a low LUMO level of $-4.24 \mathrm{eV}$, was developed as an excellent electron-accepting moiety for a large number of semiconductors in field-effect transistors (FETs). ${ }^{9}$ Specially, the D-A conjugated polymer BDOPV-2T showed a high electron mobility up to $1.74 \mathrm{~cm}^{2} \mathrm{~V}^{-1} \mathrm{~s}^{-1} \cdot{ }^{9 c}$ To further improve the electron transport property of BDOPV-based polymers, herein, we embed $\mathrm{sp}^{2}$-nitrogen atoms in BDOPV, resulting in a stronger electron-deficient building block diaza-BDOPV (AzaBDOPV) (Fig. 1). The D-A conjugated polymer AzaBDOPV2T shows a more planar backbone and a lower LUMO level (down to $-4.37 \mathrm{eV}$ ) compared with BDOPV-2T. As a consequence, AzaBDOPV-2T exhibits higher electron mobilities of over $3.22 \mathrm{~cm}^{2} \mathrm{~V}^{-1} \mathrm{~s}^{-1}$ for devices tested under ambient conditions, which is among the highest in n-type polymer FETs..$^{5 c, 10}$

To achieve the monomer AzaBDOPV, 6-bromo-7-azaisatin was synthesized from a commercial available precursor, 7-

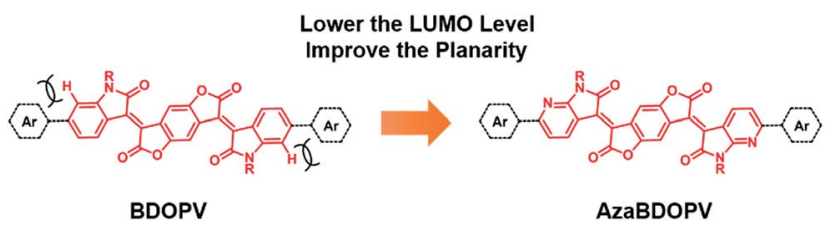

Fig. 1 Molecular structures of BDOPV and AzaBDOPV. 

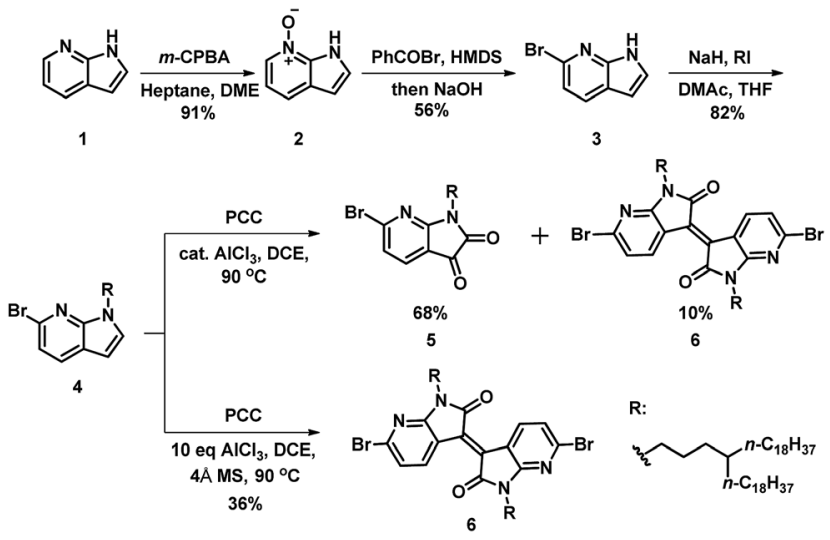

Scheme 1 Synthetic route to 6-bromo-7-azaisatin and its derivatives.

azaindole. ${ }^{11}$ As illustrated in Scheme 1, 7-azaindole (1) was converted into $N$-oxide-7-azaindole (2) by $m$-CPBA in high yield. Benzoyl bromide was used as the brominating reagent to afford a Reissert-Henze salt. ${ }^{12}$ Hence, the bromination reaction only occurred at the ortho-position relative to the nitrogen atom on pyridine. The benzoyl group on the nitrogen atom of the pyrrole was subsequently removed by the base treatment, giving 6bromo-7-azaindole (3) in 56\% yield for the two steps. Then, the alkylation of 3 gave the corresponding alkylated azaindole (4) in $82 \%$ yield, which was further oxidated through pyridinium chlorochromate (PCC) to afford 6-bromo-7-azaisatin in 68\% yield. ${ }^{13}$ Interestingly, 6,6'-dibromo-7,7'-azaisoindigo was also observed in $10 \%$ yield in this oxidation reaction. ${ }^{13 a}$ Further investigation revealed that $6,6^{\prime}$-dibromo-7, $7^{\prime}$-azaisoindigo was afforded exclusively in $36 \%$ yield when using dry PCC and excess $\mathrm{AlCl}_{3}$. The generation of azaisoindigo was expected as a result of the Lewis-acid-catalyzed aldol condensation between the azaisatin and azaindole, with subsequent dehydration and oxidation as shown in Fig. S1. $\dagger$ An excess amount of $\mathrm{AlCl}_{3}$ and a non-water environment may promote this process, resulting in selectively affording compound $6 .^{13 b}$ Considering the potential optoelectronic applications of azaisoindigo derivatives, our method provides a shorter and simpler strategy to obtain azaisoindigo compared with the more common method that requires the coupling of oxindole with isatin.

As shown in Scheme 2, AzaBDOPV was synthesized in $40 \%$ yield by a condensation reaction between 5 and benzo[1,2- $b: 4,5$ $b^{\prime}$ ]difuran-2,6 $(3 H, 7 H)$-dione in the presence of 4-methylbenzenesulfonic acid. Finally, a Stille coupling polymerization reaction between AzaBDOPV and 5,5'-bis(trimethylstannyl)-2,2'bithiophene gave the desired polymer AzaBDOPV-2T in 90\% yield after purification (see the ESI $\dagger$ ). AzaBDOPV-2T displayed a high molecular weight with an $M_{\mathrm{n}}$ of $51.6 \mathrm{kDa}$ and a polydispersity index (PDI) of 2.62, which was evaluated by high temperature gel permeation chromatography at $150{ }^{\circ} \mathrm{C}$ using 1,2,4-trichlorobenzene as the eluent (Fig. S2 $\dagger$ ). AzaBDOPV-2T also showed excellent thermal stability with a decomposition temperature of over $390{ }^{\circ} \mathrm{C}$ (Fig. S3†).

The absorption spectra of the polymer BDOPV-2T in dilute solution, and the polymer AzaBDOPV-2T both in dilute solution
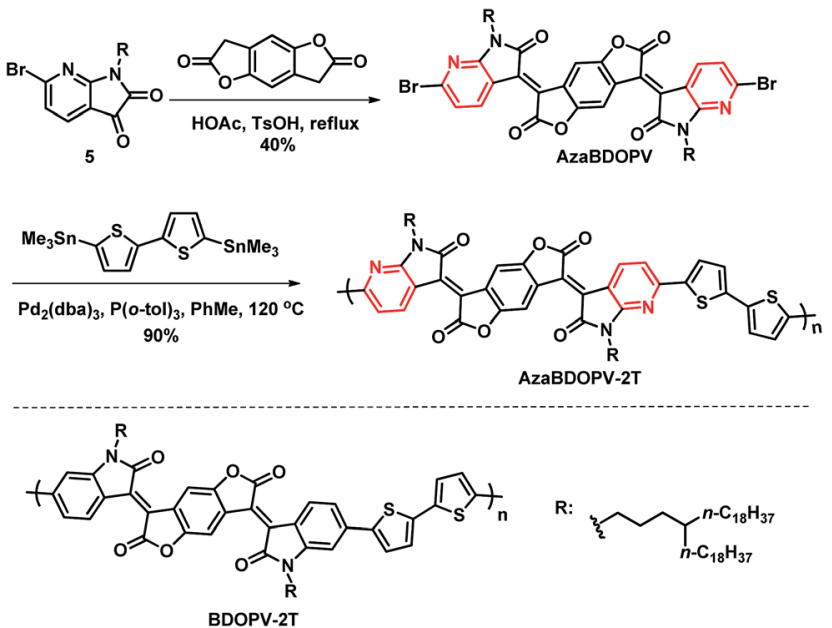

Scheme 2 Synthesis of polymer AzaBDOPV-2T and structure of the reference polymer BDOPV-2T.

and in a thin film, are shown in Fig. 2. The bandgap of AzaBDOPV-2T calculated from the onset of film absorption is 1.32 eV. AzaBDOPV-2T displayed a dual-band absorption both in solution and in film with obvious $0-0$ and $0-1$ vibrational peaks. Compared with the absorption of BDOPV-2T in dilute solution, the 0-0 vibrational peak of AzaBDOPV-2T was clearly observed in solution, indicating that AzaBDOPV-2T has a more planar backbone structure relative to BDOPV-2T. No significant redshift of the absorption was observed for AzaBDOPV-2T from the solution to the film, which indicates that the polymer probably forms some preaggregates in solution due to strong intermolecular interactions. Interestingly, computational analysis of the AzaBDOPV-2T fragment reveals that the sulfur and pyridinic nitrogen atoms were favorable to be on the same side. The calculated S-N distance $(2.27 \AA)$ is significantly shorter than the sum of the $\mathrm{S}-\mathrm{N}$ van der Waals radii (3.35 $\mathrm{A}$ ), indicating a typical S-N interaction, ${ }^{\mathbf{1 4}}$ which may "lock" the polymer conformation. As a result, the calculated pyridyl-thienyl rotational angle in AzaBDOPV-2T is decreased from $21.5^{\circ}$ to $0.5^{\circ}$, revealing that the introduction of nitrogen atoms provides the polymer with a more planar backbone and better $\pi$-conjugation, which is consistent with the absorption features.

Cyclic voltammetry (CV) was carried out to investigate the energy levels of the monomer AzaBDOPV and the polymer AzaBDOPV-2T (Fig. S4 $\uparrow$ and 3a). After the introduction of nitrogen atoms, the LUMO level of the monomer AzaBDOPV reaches $-4.35 \mathrm{eV}$, which is $0.11 \mathrm{eV}$ lower than that of BDOPV, suggesting that AzaBDOPV might be a more efficient electrondeficient building block for n-type conjugated polymers. The HOMO/LUMO levels of AzaBDOPV-2T measured from CV are $-5.80 /-4.37 \mathrm{eV}$, which are consistent with the HOMO/LUMO levels $(-5.77 /-4.45 \mathrm{eV})$ estimated from photoelectron spectroscopy (PES) (Fig. S5†) and the optical bandgap. Note that the LUMO level of AzaBDOPV-2T is also significantly lower than that of BDOPV-2T $(-4.15 \mathrm{eV})$, indicating that AzaBDOPV-2T might exhibit more efficient and stable electron transporting properties than BDOPV-2T. ${ }^{9 c}$ To our knowledge, polymer AzaBDOPV- 
(a)

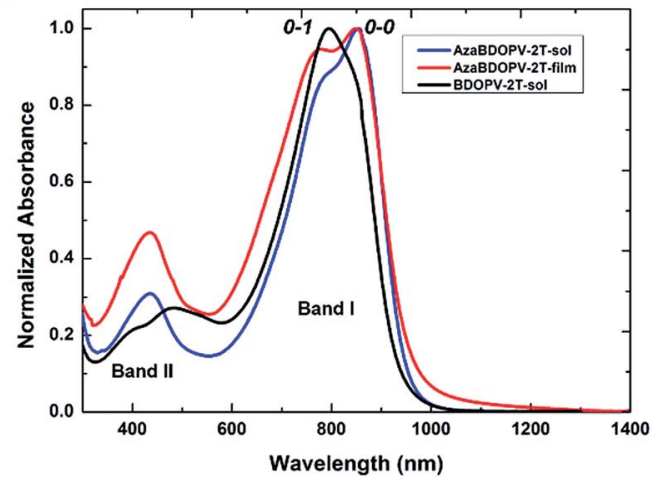

(b)

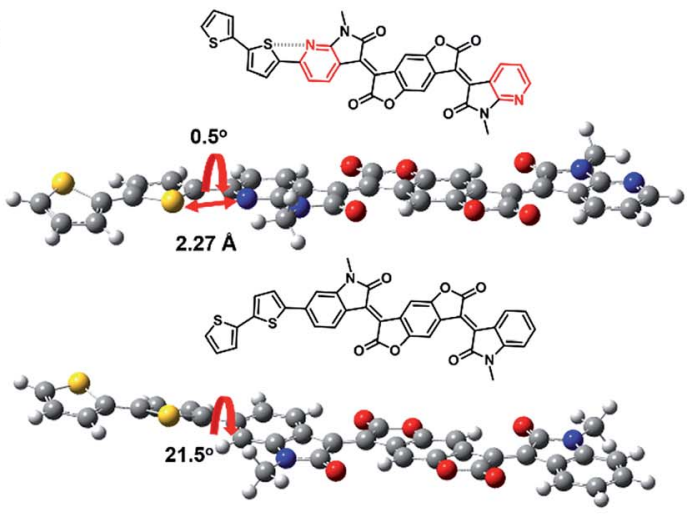

Fig. 2 (a) The absorption spectra of the polymer BDOPV-2T in dilute solution ( $\left.10^{-5} \mathrm{M}\right)$, AzaBDOPV-2T both in dilute solution (10 $\left.{ }^{-5} \mathrm{M}\right)$ and in thin film; (b) DFT-optimized geometries of AzaBDOPV-2T and BDOPV-2T fragments. Alkyl chains were replaced by methyl groups for simplicity (geometries were optimized at the b3lyp/6-311g(d,p) level).

2T is among the most electron-deficient conjugated polymers reported to date. ${ }^{15}$ The HOMO level of AzaBDOPV-2T is nearly the same as that of BDOPV-2T (HOMO level of $-5.72 \mathrm{eV}$ ), indicating that the introduction of nitrogen at the position near the amide group has larger effect on the LUMO than the HOMO. This result was in accordance with the calculated molecular orbital distribution, where the HOMO of AzaBDOPV-2T is delocalized and the LUMO is localized on the AzaBDOPV cores (Fig. 3b).

Field-effect transistors with a top-gate/bottom-contact (TG/ $\mathrm{BC})$ configuration were then fabricated to characterize the charge transport properties of AzaBDOPV-2T. The semiconducting layer was deposited by spin-coating the polymer solution $\left(3 \mathrm{mg} \mathrm{mL}{ }^{-1}\right.$ in ODCB) on an Au (source-drain)/ $/ \mathrm{SiO}_{2}$ substrate. $n$-Octane was then spin-coated onto the as-spun AzaBDOPV-2T film before thermal annealing for $5 \mathrm{~min}$. Several annealing temperatures were tried, and annealing at $200{ }^{\circ} \mathrm{C}$ gave the best device performance (Fig. S6 $\dagger$ ). After thermal annealing, a CYTOP solution was spin-coated on top of the film as the dielectric layer, and an aluminum layer was thermally evaporated as the gate electrode. All devices were fabricated in a glovebox and tested in air $\left(R_{\mathrm{H}}=50-60 \%\right)$. AzaBDOPV-2T displayed typical n-type transport characteristics (Fig. 4), with the highest electron mobility up to $3.22 \mathrm{~cm}^{2} \mathrm{~V}^{-1} \mathrm{~s}^{-1}$ (an average mobility of $1.63 \mathrm{~cm}^{2} \mathrm{~V}^{-1} \mathrm{~s}^{-1}$ from seventeen devices and the standard deviation was $0.54 \mathrm{~cm}^{2} \mathrm{~V}^{-1} \mathrm{~s}^{-1}$ ), whereas the reference polymer BDOPV-2T showed highest electron mobilities of up to $1.74 \mathrm{~cm}^{2} \mathrm{~V}^{-1} \mathrm{~s}^{-1} .^{c}$ This result is attributed to the fact that AzaBDOPV-2T has a lower LUMO energy level and less conformational disorder than BDOPV-2T.

The molecular packing and surface morphology of the AzaBDOPV-2T film were investigated by grazing incident X-ray diffraction (GIXD) and tapping-mode atomic force microscopy (AFM) as shown in Fig. 5 and S7. $\dagger$ The film of AzaBDOPV-2T showed a strong out-of-plane diffraction peak (100) at a $2 \theta$ value of $2.50^{\circ}$, corresponding to a $d$-spacing of $28.4 \AA(\lambda=1.24 \AA)$. Another four orders of diffraction peaks attributed to (h00) diffractions and an in-plane diffraction peak of (010) was also observed, indicating a distinct edge-on lamellar packing formed in the film. After the introduction of nitrogen atoms, the $\pi-\pi$ stacking distance of AzaBDOPV-2T was measured to be $3.44 \AA$ (Fig. S8 $\dagger$ ), which was shorter than that of BDOPV-2T (3.55 $)$ ), suggesting stronger inter-chain interactions of AzaBDOPV-2T in the solid state compared to BDOPV-2T. The strong crystallinity of AzaBDOPV-2T is also supported by the AFM image, which displays a fiber-like intercalating network with obvious crystallized zones. Both the close $\pi-\pi$ stacking distance and strong crystallinity are beneficial to the high mobilities of AzaBDOPV-2T. (a)

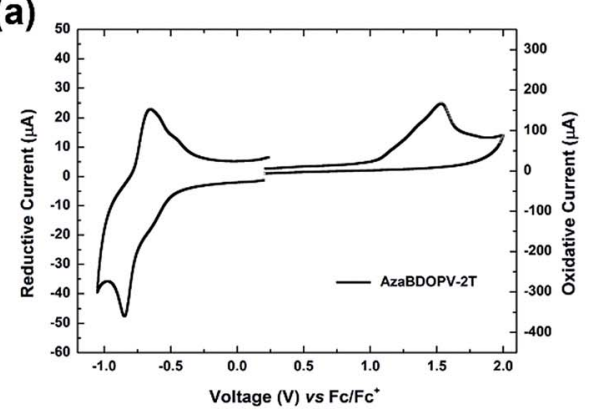

(b)

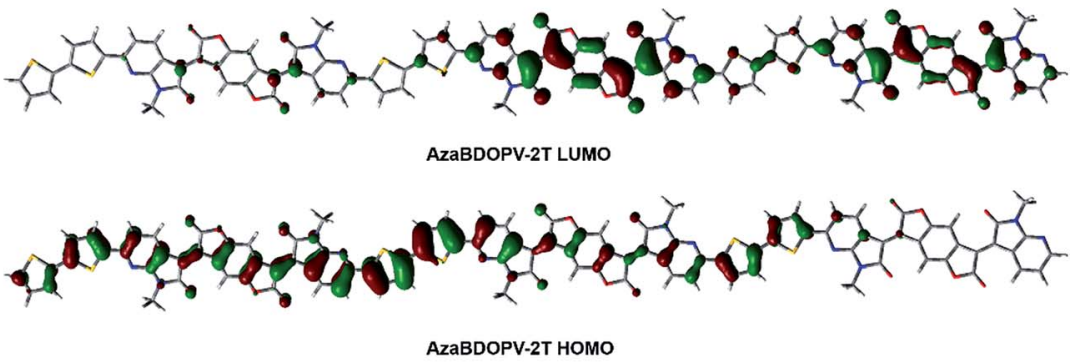

Fig. 3 (a) Cyclic voltammogram of the polymer AzaBDOPV-2T in thin film prepared by drop-casting the polymer solution (1 $\mathrm{mg} \mathrm{mL}^{-1}$ in $\mathrm{CHCl}_{3}$ ) on a glassy carbon electrode; (b) calculated molecular orbitals of the AzaBDOPV-2T trimer at the b3lyp/6-31g(d) level. 
(a)

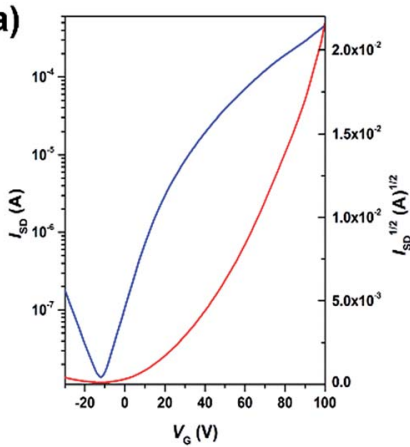

(b)

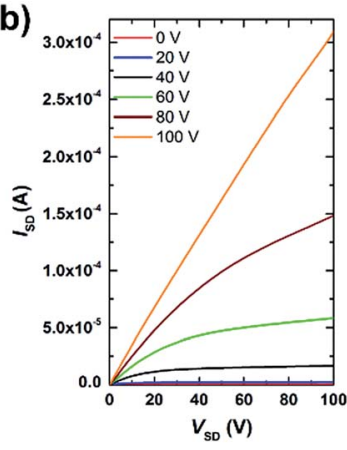

Fig. 4 (a) Transfer and (b) output characteristics of an AzaBDOPV-2T device tested under ambient conditions $\left(L=5 \mu \mathrm{m}, W=100 \mu \mathrm{m}, C_{i}=\right.$ $3.5 \mathrm{nF} \mathrm{cm}^{-2}$ ), measured $V_{T}=40 \mathrm{~V}$.
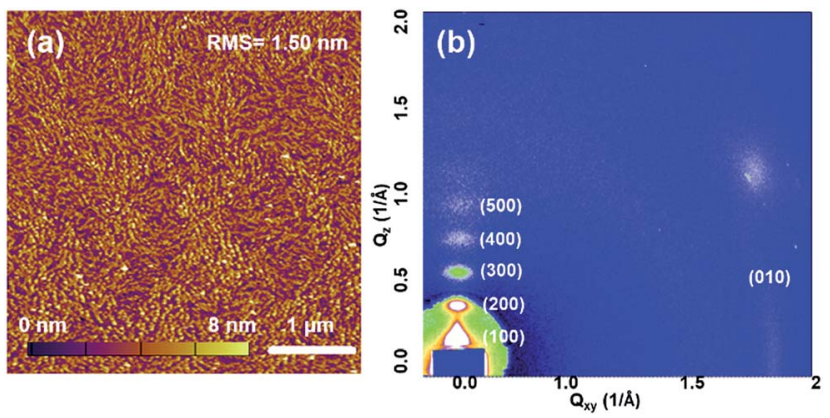

Fig. 5 (a) AFM height image and (b) 2D-GIXD pattern of AzaBDOPV2T film spin-coated on a $\mathrm{SiO}_{2}$ substrate after thermal annealing at 200 ${ }^{\circ} \mathrm{C}$ for $5 \mathrm{~min}$.

\section{Conclusions}

In conclusion, we have developed a novel electron-deficient building block, AzaBDOPV, which shows a much lower LUMO level compared with BDOPV. Based on the AzaBDOPV unit, the D-A conjugated polymer, AzaBDOPV-2T, displays a low LUMO level down to $-4.37 \mathrm{eV}$ and therefore typical n-type transport characteristics, with electron mobilities up to $3.22 \mathrm{~cm}^{2} \mathrm{~V}^{-1} \mathrm{~s}^{-1}$ in air. These investigations demonstrate that the incorporation of electron-withdrawing $\mathrm{sp}^{2}$-nitrogen atoms into a BDOPVbased polymer not only lowers the energy levels of the conjugated polymer, but also optimizes its backbone conformation, leading to improved inter-chain interactions and film microstructures, which are critical to achieving high device performance.

\section{Acknowledgements}

This work was supported by the Major State Basic Research Development Program (No. 2013CB933501 and 2015CB856505) from the Ministry of Science and Technology, National Natural Science Foundation of China. The authors thank beamline BL14B1 (Shanghai Synchrotron Ra-diation Facility) for providing the beam time.

\section{Notes and references}

1 (a) A. J. Heeger, Chem. Soc. Rev., 2010, 39, 2354; (b) J. Mei, Y. Diao, A. L. Appleton, L. Fang and Z. Bao, J. Am. Chem. Soc., 2013, 135, 6724; (c) C. Wang, H. Dong, W. Hu, Y. Liu and D. Zhu, Chem. Rev., 2012, 112, 2208; (d) Y.-Q. Zheng, J.-Y. Wang and J. Pei, Sci. China: Chem., 2015, 58, 937.

2 (a) S. Wang, M. Kappl, I. Liebewirth, M. Müller, K. Kirchhoff, W. Pisula and K. Müllen, Adv. Mater., 2012, 24, 417; (b) H. N. Tsao, D. M. Cho, I. Park, M. R. Hansen, A. Mavrinskiy, D. Y. Yoon, R. Graf, W. Pisula, H. W. Spiess and K. Müllen, J. Am. Chem. Soc., 2011, 133, 2605; (c) H.-R. Tseng, L. Ying, B. B. Y. Hsu, L. A. Perez, C. J. Takacs, G. C. Bazan and A. J. Heeger, Nano Lett., 2012, 12, 6353.

3 (a) R. S. Ashraf, I. Meager, M. Nikolka, M. Kirkus, M. Planells, B. C. Schroeder, S. Holliday, M. Hurhangee, C. B. Nielsen, H. Sirringhaus and I. McCulloch, J. Am. Chem. Soc., 2015, 137, 1314; (b) J. Li, Y. Zhao, H. S. Tan, Y. Guo, C.-A. Di, G. Yu, Y. Liu, M. Lin, S. H. Lim, Y. Zhou, H. Su and B. S. Ong, Sci. Rep., 2012, 2, 754; (c) C. B. Nielsen, M. Turbiez and I. McCulloch, Adv. Mater., 2013, 25, 1859; (d) Z. Yi, S. Wang and Y. Liu, Adv. Mater., 2015, 27(3589), 1; (e) Y. Ji, C. Xiao, G. H. L. Heintges, Y. Wu, R. A. J. Janssen, D. Zhang, W. Hu, Z. Wang and W. Li, J. Polym. Sci., Part A: Polym. Chem., 2016, 54, 34.

4 (a) R. Stalder, S. R. Puniredd, M. R. Hansen, U. Koldemir, C. Grand, W. Zajaczkowski, K. Müllen, W. Pisula and J. R. Reynolds, Chem. Mater., 2016, 28, 1286; (b) R. Stalder, J. Mei, K. R. Graham, L. A. Estrada and J. R. Reynolds, Chem. Mater., 2014, 26, 664; (c) T. Lei, Y. Cao, Y. Fan, C.-J. Liu, S.-C. Yuan and J. Pei, J. Am. Chem. Soc., 2011, 133, 6099; (d) J. Mei, D. H. Kim, A. L. Ayzner, M. F. Toney and Z. Bao, J. Am. Chem. Soc., 2011, 133, 20130.

5 (a) H. Huang, Z. Chen, R. P. Ortiz, C. Newman, H. Usta, S. Lou, J. Youn, Y.-Y. Noh, K.-J. Baeg, L. X. Chen, A. Facchetti and T. Marks, J. Am. Chem. Soc., 2012, 134, 10966; (b) K.-J. Baeg, D. Khim, S.-W. Jung, M. Kang, I.-K. You, D.-Y. Kim, A. Facchetti and Y.-Y. Noh, Adv. Mater., 2012, 24, 5433; (c) B. Kang, R. Kim, S. B. Lee, S. K. Kwon, Y. H. Kim and K. Cho, J. Am. Chem. Soc., 2016, 138, 3679.

6 (a) J. Zaumseil and H. Sirringhaus, Chem. Rev., 2007, 107, 1296; (b) R. Di Pietro, D. Fazzi, T. B. Kehoe and H. Sirringhaus, J. Am. Chem. Soc., 2012, 134, 14877; (c) Z. Fei, P. Boufflet, S. Wood, J. Wade, J. Moriarty, E. Gann, E. L. Ratcliff, C. R. McNeill, H. Sirringhaus, J. S. Kim and M. Heeney, J. Am. Chem. Soc., 2015, 137, 6866; (d) T. Lei, J.-H. Dou, Z.-J. Ma, C.-J. Liu, J.-Y. Wang and J. Pei, Chem. Sci., 2013, 4, 2447; (e) X.-K. Gao and Y.-B. Hu, J. Mater. Chem. C, 2014, 2, 3099; (f) Z. Zhao, F.-J. Zhang, Y.-B. Hu, Z.-L. Wang, B. Leng, X.-K. Gao, C.-A. Di and D.-B. Zhu, ACS Macro Lett., 2014, 3, 1174.

7 (a) J. Huang, Z. Mao, Z. Chen, D. Gao, C. Wei, W. Zhang and G. Yu, Chem. Mater., 2016, 28, 2209; (b) T. L. D. Tam and T. T. Lin, Macromolecules, 2016, 49, 1648; (c) J. Wang, 
X. Chen, Z. Cai, H. Luo, Y. Li, Z. Liu, G. Zhang and D. Zhang, Polym. Chem., 2013, 4, 5283.

8 B. Sun, W. Hong, Z. Yan, H. Aziz and Y. Li, Adv. Mater., 2014, 26, 2636.

9 (a) Z. Yan, B. Sun and Y. Li, Chem. Commun., 2013, 49, 3790; (b) T. Lei, J.-H. Dou, X.-Y. Cao, J.-Y. Wang and J. Pei, J. Am. Chem. Soc., 2013, 135, 12168; (c) T. Lei, J.-H. Dou, X.-Y. Cao, J.-Y. Wang and J. Pei, Adv. Mater., 2013, 25, 6589.

10 T. Lei, X. Xia, J. Y. Wang, C. J. Liu and J. Pei, J. Am. Chem. Soc., 2014, 136, 2135.

11 During our preparation of this manuscript, Yu et al. reported the synthesis of diazaisoindigo based polymers from 6bromo-7-azaisatin (see ref. 7a), which was synthesized according to the literature method. Herein, we adopt an optimized reaction which was performed by a simplified procedure and could obtain diazaisoindigo exclusively in appropriate yield.

12 M. K. Satoshi Minakata and Y. Ohshiro, Synthesis, 1992, 661. 13 (a) R. Sriram, C. N. Sesha Sai Pavan Kumar, N. Raghunandan, V. Ramesh, M. Sarangapani and V. J. Rao, Synth. Commun., 2012, 42, 3419; (b) P. S. Prathima, P. Rajesh, J. V. Rao, U. S. Kailash, B. Sridhar and M. M. Rao, Eur. J. Med. Chem., 2014, 84, 155. 14 R. Ghosh and S. H. Simonsen, Acta Crystallogr., Sect. C: Cryst. Struct. Commun., 1993, 49, 1031.

15 (a) J. D. Yuen and F. Wudl, Energy Environ. Sci., 2013, 6, 392; (b) Y. Wen, Y. Liu, Y. Guo, G. Yu and W. Hu, Chem. Rev., 2011, 111, 3358; (c) X. Zhang, C. Xiao, A. Zhang, F. Yang, H. Dong, Z. Wang, X. Zhan, W. Li and W. Hu, Polym. Chem., 2015, 6, 4775. 